\title{
Supporting the need for community exercise programs: a case study
}

\author{
Christopher Repecki ${ }^{1}$ Martha Sliwinski ${ }^{1}$ Lawrence Harding ${ }^{2}$
}

Received: 17 March 2019 / Revised: 3 September 2019 / Accepted: 11 October 2019

(c) International Spinal Cord Society 2019

\begin{abstract}
Introduction Participation in exercise activities post spinal cord injury (SCI) can positively impact physical functioning and quality of life. Physically active individuals have improved functional performance compared with sedentary individuals with SCI. Consistent exercise interventions following SCI not only prompt neural recovery and offer myriad health benefits but they may also have persisting effects on functional abilities.

Case presentation A 29-year-old male subject had a 15-year history of a C5-C6 AIS B SCI. The subject demonstrated improvements in the outcome measures and he reported enhanced ability to cough and to clear secretions, as well as an enhanced overall quality of life, after undergoing a 14-week course of Spinal Mobility training in combination with inspiratory muscle training (IMT). In addition to the Spinal Mobility training and IMT, he continued to partake in his normal exercise routine, which consisted of aerobic and strength training 3 days per week.

Discussion This case reaffirms the benefits of consistent exercise training, in combination with IMT, for individuals with SCI. Community programs specifically targeted to individuals with SCI are needed. The subject's functional improvements demonstrate that incorporating Spinal Mobility training into a regular exercise routine may assist with functional gains in the chronic stage of SCI.
\end{abstract}

\section{Introduction}

Exercise training that incorporates aerobic and strength training components has the potential to significantly reduce secondary morbidities associated with SCI. Physical activity has been shown to not only reduce many of these secondary health complications but it has also been positively correlated with improvements in strength, endurance, and functional ability [1-3]. Previous literature has found a correlation between increased physical activity and healthrelated improvements in individuals with a SCI; namely, overall less pathology and comorbidities have been demonstrated in more physically active individuals [1]. Respiratory compromise is also common in individuals with SCI, particularly with injuries lacking abdominal innervation that can compromise both inspiratory and expiratory neuromuscular control. IMT can provide added benefits to

Christopher Repecki

chrisrepecki7@gmail.com

1 Columbia University Medical Center, Program in Physical Therapy, New York, NY, USA

2 The Axis Project, 1325 5th Ave, New York, NY, USA regular exercise in this population $[2,4,5]$. A progressive resistive load can be applied to the diaphragm, intercostal, and accessory muscles to improve lung volumes and blood pressure regulation [6-8].

Despite the benefits and need for exercise after SCI in individuals with chronic injuries, there is no insurance coverage for individuals in the US for these activities. In addition, there is a lack of funding for community-based programs. In order to facilitate continued exercise, the Spinal Mobility X class (SMX), a community-based exercise program, was developed. It has shown a positive effect on function and quality of life for people with SCI [9]. The Spinal Mobility technique consists of exercise interventions to improve trunk stabilization control, geared toward improving function, mobility, and quality of life in individuals with neurologically based disability in all phases of the rehabilitation process. The technique is grounded in theories of neuroplasticity, motor learning, and biomechanics, incorporating elements of segmental stability, distal locking, and reverse muscle activity. These training components are transferable to the trunk mobility and stability requirements necessary for daily activities, such as transfers, grooming, bathing, reaching, and community mobility. This case report describes the impact of a 14-week 
course of the Spinal Mobility technique, along with IMT, on an individual in the chronic stages of SCI $[1-3,10]$.

\section{Case presentation}

A 29-year-old male had a 15-year history of a C5-C6 AIS B SCI. The subject works full-time, and he uses a power wheelchair as his primary means of mobility. He lives an active lifestyle, and he has engaged in strength and aerobic exercise on a consistent basis for many years. Prior to undergoing a 14-week course of Spinal Mobility training, the subject reported difficulties performing transfers, bed mobility, general self-care, activities of daily living, and the ability to cough and to clear secretions. His normal exercise regimen included an aerobic and strength training routine, 3 days per week. He continued this exercise routine throughout the entire 14-week Spinal Mobility training period. Throughout the study period, the subject selfcatheterized and he had no changes in his bowel/bladder routine or any changes in diet or medication.

The Spinal Mobility X (SMX) class is a community-based exercise program specifically designed for individuals with SCI. The program consists of three circuits: $1 \mathrm{~h}$ of resistive activities, $1 \mathrm{~h}$ of aerobic activities, and $1 \mathrm{~h}$ of Spinal Mobility activities, all targeted toward improving control of trunk musculature and enhancing functional balance in people with SCI. The Spinal Mobility technique consists of three principles: segmental mobility, distal support, and enhanced neuromuscular control (reverse muscle activity). The segmental mobility technique is the mechanism by which the use of a mobilization belt is placed on the patient's trunk and attached to the therapist, thus allowing the therapist to provide a stable base of support and enabling the patient to regain greater trunk control above the belt location. The belt provides sensory input, and its level of placement on the trunk allows for variability in the degree of trunk control required during movement patterns: the lower the belt is placed, the greater the demands on the patient for postural control during movement patterns. Simultaneously, this technique incorporates upper quarter concentric or eccentric muscle activity during which the therapist is able to vary the overall quantity of work that the patient performs. When applying the principle of distal support, the patient is able to relearn how to control the limits of stability of their trunk, thereby enhancing their control over movements of the opposite upper extremity. This technique simulates daily tasks where individuals stabilize with one upper extremity to allow for increased trunk excursion to gain further reach with the opposite extremity. Finally, neuromuscular control, reverse muscle activity, is improved as individuals relearn how to use trunk movements assisted by intact distal musculature movement patterns to enhance proximal trunk control. For example, an individual in a manual wheelchair can use a doorframe to assist in pushing themselves through an entrance. Training in a seated position allows the patient to operate in a more biomechanically and physiologically efficient manner because they do not have to rely on lower extremity function for stability. This technique can be applied to individuals with complete or incomplete cervical, thoracic and/or lumbar injuries.

The interventions for this case consisted of once weekly Spinal Mobility group training for 8-week duration. This training included aerobic and strength training components, along with the Spinal Mobility technique. This 8-week period was followed by 6 weeks of additional one-on-one Spinal Mobility training sessions for $1 \mathrm{~h}$, twice per week. Functional outcome measures included a timed t-shirt test, timed transfer to and from a mat table, and functional reach in a forward, left and right lateral direction. Inspiratory muscle training (IMT) was added at home for the 14-week period, which the subject reportedly discontinued after all Spinal Mobility training ended. A subjective questionnaire was administered at the start and conclusion of the study to track the subject's perceived improvements in functional capacity and overall quality of life.

Outcome measures were taken 4 weeks prior to the initiation of spinal mobility interventions (time point one) and at final post testing 10 weeks after cessation of interventions (time point two). The subject was instructed to keep an IMT training diary of his home training on the daily $\log$ sheets provided for him throughout the training period, which he failed to turn in at the conclusion of the study.

The timed $t$-shirt test measured how fast the subject could put on and take off a t-shirt. The tester started the timer when the subject's hand made contact with the t-shirt placed on the adjacent table. The tester stopped the timer once the subject had completely taken off the t-shirt. Timed transfer to and from a mat table timed how fast the subject could transfer from his wheelchair to lying supine on a mat table, and then transfer back into a seated position in his wheelchair. The tester started the timer once the subject initiated the transfer, and stopped the timer once the subject had returned to a seated position in the wheelchair. The functional reach in forwards, left, and right lateral direction was measured with a yardstick taped to the wall. For the forward reach, the subject sat in his wheelchair parallel to the yardstick and reached forward as far as his limits of stability would allow. For the right and left lateral reach, the subject stationed his wheelchair perpendicular to the yardstick and reached as far as possible to the sides in both directions.

\section{Discussion}

Our case report demonstrates the beneficial effects of exercise training, specifically the Spinal Mobility technique 
in combination with IMT, in this individual with chronic SCI. The results between time point one and time point two, 10 weeks after the termination of the intervention protocol, demonstrated improvements in all measures with the exception of forward and left lateral reach. The timed t-shirt test was performed $13 \mathrm{~s}$ faster ( $43.33 \%$ improvement). Our subject's transfer test time was $16.67 \mathrm{~s}$ faster $(16.18 \%$ improvement), and right lateral reach increased 1.70 inches (50.15\% improvement) (Table 1).

Studies have demonstrated that improvements with dynamic postural control are prerequisites for independent functional performance $[11,12]$. Seated activities of daily living require dynamic postural trunk control for independence. As dynamic postural control improves, performance in activities of daily living (ADL) tasks becomes more efficient [13]. Maximal forward reach distance has been demonstrated to be a valid measure of postural control in individuals with SCI [13, 14]. The subject's improvements in the t-shirt test and right lateral reach test may be secondary to enhanced dynamic seated postural control [12]. It has been shown that reaching tasks will be a challenge to upright control for individuals with paralysis of the trunk or arms [14]. In order to successfully perform ADLs in a seated position, one must maintain trunk stability and mobility without a loss of balance [11]. The goal of the Spinal Mobility technique is to improve an individual's ability to control the trunk, which ultimately helps facilitate arm movement without a loss of balance for safe functional activities. The seated reach test is a valid measure of seated postural control, and previous research has demonstrated a correlation between maximal forward reach distance and scores on clinical tests of function in individuals with SCI [13]. Dynamic postural control is related to dynamic trunk excursion [13]. Right lateral reach demonstrated the greatest improvement (1.70 inches); this gain may in part contribute to why the patient reported improvements in ADL. The lack of improvement in the left lateral reach might be explained partly by the fact that the subject is right handed and he therefore does not engage in reaching tasks to the left very often throughout his daily life [13].

The participant reported increased ease of transfers, enhanced overall quality of life, improved ability to cough and to clear secretions, and augmented functional mobility post training. These objective and subjective findings demonstrate the benefits of exercise training, in combination with IMT, in our subject. Importantly, transfer time and $\mathrm{t}$-shirt test improvements, which are both critical components for efficiency with daily tasks and independence with ADLs, persisted after a 10-week period without any spinal mobility training. The timed t-shirt test has proven reliability and validity in SCI, and it serves as an
Table 1 Pre and post test measures

\begin{tabular}{lrrr}
\hline Pre and post test measures & Test 1 & Test 2 & \% Change \\
\hline Forward reach (in) & 6.60 & 6.30 & $-4.10 \%$ \\
Sideways reach right (in) & 3.30 & 5.00 & $50.15 \%$ \\
Sideways reach left (in) & 3.00 & 2.30 & $-22.33 \%$ \\
Timed t-shirt test (s) & 30.00 & 17.00 & $-43.33 \%$ \\
Timed transfer test (s) & 103.00 & 86.30 & $-16.18 \%$ \\
\hline
\end{tabular}

indicator of an individual's ability to sit unsupported [13]. The ability to sit unsupported is essential for people with SCIs, as many functional activities are performed from this position.

A sedentary lifestyle contributes to further deconditioning, increased dependency, and decreased overall quality of life in this population [15]. This case report supports previous literature that there is an enduring need for community organizations for health promotion and education, especially considering the changing health care system that shortens length of inpatient hospitalization. However, these community organizations are unfortunately lacking and underfunded through medical insurance. In addition, because a major goal of many individuals with SCI involves improving strength and fitness, community organizations that offer lifetime services are necessary [16]. These community organizations have the potential to increase function and improve overall health in people with SCI.

This is only a case report of an active individual with a chronic SCI; thus we cannot say if the Spinal Mobility training and IMT would provide similar benefits in someone who is not physically active or is acutely post injury. However, we believe the improvements in functional outcome measures from pre-test to post test were related to the Spinal Mobility training and IMT and that this highlights the need for future research to explore the value of Spinal Mobility training and IMT in a larger population.

Acknowledgements We thank our subject who volunteered to participate in this study. We also thank Lawrence Harding and the members of the Axis Project Harlem for their assistance in this study.

\section{Compliance with ethical standards}

Conflict of interest The authors declare that they have no conflict of interest.

Ethical approval We certify that all applicable institutional and governmental regulations concerning the ethical use of human volunteers were followed during the course of this research.

Publisher's note Springer Nature remains neutral with regard to jurisdictional claims in published maps and institutional affiliations. 


\section{References}

1. Montesinos-Magraner L, Serra-Ano P, Garcia-Masso X, RamirezGarceran L, Gonzalez LM, Gonzalez-Viejo MA. Comorbidity and physical activity in people with paraplegia: a descriptive crosssectional study. Spinal Cord 2018;56:52-6.

2. Bochkezanian V, Raymond J, de Oliveira CQ, Davis GM. Can combined aerobic and muscle strength training improve aerobic fitness, muscle strength, function and quality of life in people with spinal cord injury? A systematic review. Spinal Cord 2015;53:418-31.

3. van der Scheer JW, Martin Ginis KA, Ditor DS, Goosey-Tolfrey VL, Hicks AL, West CR, et al. Effects of exercise on fitness and health of adults with spinal cord injury: a systematic review. Neurology 2017;89:736-45.

4. Hicks AL, Martin KA, Ditor DS, Latimer AE, Craven C, Bugaresti J, et al. Long-term exercise training in persons with spinal cord injury: Effects on strength, arm ergometry performance and psychological well-being. Spinal Cord 2003;41:34-43.

5. Berlowitz DJ, Tamplin J. Respiratory muscle training for cervical spinal cord injury. Cochrane Database of Systematic Reviews. 2013;7:1-36.

6. Mueller G, Hopman MT, Perret C. Comparison of respiratory muscle training methods in individuals with motor and sensory complete tetraplegia: a randomized controlled trial. J Rehabil Med. 2013;45:248-53.

7. Aslan SC, Randall DC, Krassioukov AV, Phillips A, Ovechekin AV. Respiratory training improves blood pressure regulation in individuals with chronic spinal cord injury. Arch Phys Med Rehabil. 2016;97:964-73.
8. Kellens I, Cannizzaro F, Gouilly P, Crielaard JM. Inspiratory muscles strength training in recreational athletes. Rev des Maladies Respiratoires. 2011;28:602-8.

9. Sliwinski MM, Akselrad G, Alla V, Buan V, Kaemmerlen E. Community exercise programing and its potential influence on quality of life and functional reach for individuals with spinal cord injury. J Spin Cord Med. 2018. https://doi.org/10.1080/10790268. 2018.1543104.

10. Savic G, DeVivio MJ, Frankel HL, Jamous MA, Soni BM, Charlifue S. Long-term survival after traumatic spinal cord injury: a 70-year British Study. Spinal Cord. 2017;55:651-8.

11. Chen CL, Yeung KT, Bih LI, Wang CH, Chen MI, Chien JC. The relationship between sitting stability and functional performance in patients with paraplegia. Arch Phys Med Rehabil. 2003;84:1276-81.

12. Sprigle S, Maurer C, Holowka M. Development of valid and reliable measures of postural stability. J Spinal Cord Med. 2007;30:40-9.

13. Field-fote EC, Ray SS. Seated reach distance and trunk excursion accurately reflect dynamic postural control in individuals with motor-incomplete spinal cord injury. Spinal Cord. 2010;48:745-9.

14. Lynch SM, Leahy P, Barker SP. Reliability of measurements obtained with a modified functional reach test in subjects with spinal cord injury. Phys Ther. 1998;78:128-33.

15. Boswell-ruys CL, Harvey LA, Barker JJ, Ben M, Middleton JW, Lord SR. Training unsupported sitting in people with chronic spinal cord injuries: a randomized controlled trial. Spinal Cord. 2010;48:138-43.

16. Jörgensen S, Martin ginis KA, Lexell J. Leisure time physical activity among older adults with long-term spinal cord injury. Spinal Cord. 2017;55:848-56. 